\title{
ORIGINAL ARTICLE \\ Burden of spinal cord injury-related neuropathic pain in the United States: retrospective chart review and cross-sectional survey
}

\author{
R Mann ${ }^{1}$, C Schaefer ${ }^{2}$, A Sadosky ${ }^{3}$, F Bergstrom ${ }^{2}$, R Baik ${ }^{2}$, B Parsons ${ }^{3}$, S Nalamachu ${ }^{4}$, BR Stacey ${ }^{5}$, \\ M Tuchman ${ }^{6}$, A Anschel ${ }^{7}$ and EC Nieshoff ${ }^{8}$
}

\begin{abstract}
Study design: Cross-sectional, observational study.
Objectives: Characterize demographic and clinical characteristics, health status, pain, function, productivity and economic burden in spinal cord injury-related neuropathic pain (SCl-NeP) subjects, by pain severity.

Setting: United States.

Methods: One hundred and three subjects diagnosed with $\mathrm{SCl}-\mathrm{NeP}$ recruited during routine primary care or specialty physician office visits completed a questionnaire to assess patient-reported outcomes. Physicians completed a case report form on inclusion/exclusion criteria, subject clinical characteristics and health-care resource use (HRU) based on 6-month retrospective chart review.

Results: Subjects' mean age was $48.7,69.9 \%$ were male and $48.5 \%$ were unable to walk. The most frequently reported comorbidities were sleep disturbance/insomnia (28.2\%), depressive symptoms (25.2\%) and anxiety (23.3\%). Subjects' mean pain severity score was 5.3 (0-10 scale), and $77.7 \%$ reported moderate or severe pain. On a 0-10 scale, subjects' reported moderate pain interference with function: mean 5.4. Subjects' health status, as measured by the EuroQol 5-dimensions health-state utility, was 0.49 $(-0.11$ to 1.00 scale). Pain interference with function and health status were significantly worse among subjects with more severe pain $(P<0.0005)$. Among employed subjects $(13.6 \%)$, overall work impairment was $38.0 \%$. The proportion of subjects who were prescribed $\geqslant 1$ medication was $94.2 \%$, and the mean number of physician office visits in past 6 months due to SCl-NeP was 2.2. Total annualized cost per subject was $\$ 26270$ (direct: $\$ 8636$, indirect: $\$ 17634$ ).

Conclusion: SCI-NeP subjects exhibited high pain levels, despite active management. Pain levels were associated with poor function, low health status and lost productivity. HRU was prevalent, and costs, particularly indirect, were substantial, highlighting unmet need. Sponsorship: This study was supported by Pfizer, Inc.
\end{abstract}

Spinal Cord (2013) 51, 564-570; doi:10.1038/sc.2013.34; published online 16 April 2013

Keywords: spinal cord injury-related neuropathic pain; burden of illness; quality-of-life; health-care resource use; costs; productivity

\section{INTRODUCTION}

Recent research indicates that up to $50 \%$ of individuals with spinal cord injury (SCI) develop neuropathic pain (NeP). ${ }^{1}$ Although the mechanisms underlying SCI-related NeP (SCI-NeP) are not fully understood, SCI-NeP is characterized by spontaneous persistent pain and described as abnormal sensations, including burning, tingling, stabbing, shooting and aching. ${ }^{2}$

Several US studies have evaluated the impact of post-SCI pain on patient-reported outcomes. ${ }^{3-5}$ Raichle et al..$^{3}$ found that as pain severity increased among subjects with post-SCI pain, pain interference with function and disability increased. In another study, SCI patients reported high pain intensity and moderate pain interference with activities, and rated pain treatments received as only somewhat helpful. ${ }^{4}$ Widerstrom-Noga et al. ${ }^{5}$ found that individuals experiencing high-intensity post-SCI pain were more likely than others to experience frequent interference with daily activities, including sleep.
To our knowledge, although an important component of post-SCI pain, no published studies have assessed patient-reported outcomes, health-care resource use (HRU) or costs in SCI-NeP specifically. A more comprehensive assessment of the burden of SCI-NeP could inform physicians of unmet needs in this population and lead to more comprehensive clinical management. Therefore, the study objective was to characterize SCI-NeP burden by pain severity level by assessing sociodemographic and clinical characteristics; impact of SCI-NeP on anxiety, depression, sleep, health status, productivity, current treatment patterns and HRU, and direct and indirect costs.

\section{MATERIALS AND METHODS}

This cross-sectional, observational study recruited SCI-NeP subjects between September 2011 and June 2012 from 14 community-based US physician practices, including 2 general practitioners, 5 neurologists, 5 pain specialists, 1 physiatrist and 1 rheumatologist.

${ }^{1}$ Covance Market Access Services Inc., San Diego, CA, USA; ${ }^{2}$ Covance Market Access Services Inc., Gaithersburg, MD, USA; ${ }^{3}$ Health Economics and Outcomes Research, Pfizer, Inc., New York, NY, USA; ${ }^{4}$ International Clinical Research Institute, Overland Park, KS, USA; ${ }^{5}$ Oregon Health andScience University, Portland, OR, USA; ${ }^{6}$ Palm Beach Neurological Center, Palm Beach Gardens, FL, USA; ${ }^{7}$ Rehabilitation Institute of Chicago, Chicago, IL, USA and ${ }^{8}$ Rehabilitation Institute of Michigan/Wayne State University, Detroit, MI, USA

Correspondence: Dr A Sadosky, Health Economics and Outcomes Research, Pfizer Inc., 235 East 42nd Street, New York, NY 10017, USA.

E-mail: alesia.sadosky@pfizer.com

Received 16 November 2012; revised 28 February 2013; accepted 16 March 2013; published online 16 April 2013 
A central Institutional Review Board, Concordia Clinical Research (Cedar Knolls, NJ), approved the study.

SCI-NeP was defined as: (1) SCI (complete or incomplete paraplegia or tetraplegia) of $\geqslant 1$ year duration with a non-progressive (chronic) stage of $\geqslant 6$ months duration and (2) NeP, starting after the SCI and persisting for $\geqslant 3$ continuous months or with remissions and relapses for $\geqslant 6$ months. As SCI$\mathrm{NeP}$ patients presented for routine office visits, a trained physician or site coordinator identified and recruited subjects. Interested subjects provided informed consent before data collection.

Adult subjects ( $\geqslant 18$ years) diagnosed with SCI-NeP and managed at their physician's practice for $\geqslant 6$ months were eligible for enrollment. Subjects were required to read and understand English. Subjects were not eligible if they participated in an investigational drug study in the 6 months before enrollment, had a serious or unstable medical or psychological condition that would compromise participation in the study or had a concomitant illness unrelated to SCI-NeP that may confound the assessment of SCI-NeP.

\section{Data collection}

Enrolled subjects completed a one-time self-administered questionnaire, including questions on demographics, NeP symptom duration, SCI-NeP non-prescription treatments, out-of-pocket costs in the past 4 weeks, employment status and patient-reported outcome measures: the Brief Pain InventoryShort Form (BPI-SF), ${ }^{6}$ 12-item Short-Form Health Survey (SF-12v2; 1-week recall), ${ }^{7}$ EuroQol 5-dimensions, 3-levels (EQ-5D-3L), ${ }^{8}$ Medical Outcomes Study Sleep Scale (MOS-SS), ${ }^{9}$ Hospital Anxiety and Depression Scale $(\text { HADS })^{10,11}$ and Work Productivity and Activity Impairment (WPAI) due to SCI-NeP. ${ }^{12}$

The participating physician or site coordinator reviewed the subject's medical chart for SCI-NeP diagnosis date, duration of SCI, comorbidities, SCI-NeP prescription treatments and SCI-NeP HRU over the past 6 months.

The BPI-SF included a four-item measure of pain severity (worst, least, average and current) and seven-item measure of pain interference with function. ${ }^{6}$ Items were assessed on 11-point numeric rating scales $(0=$ no pain to $10=$ pain as bad as you can imagine).

The SF-12v2 contained 12 items assessing eight domains. Composite physical and mental component scores were calculated (0-100 scale). Higher scores indicated better outcomes.

The EQ-5D is a five-item health status and utility measure. ${ }^{8}$ Health-state valuation scores range from -0.11 to 1.00 . Higher scores indicated better health status.

The MOS-SS included 12 questions $^{9}$ with nine items comprising the Sleep Problems Index (0-100 scale). Higher scores indicated worse outcomes.

The HADS is a 14-item measure consisted of anxiety and depression 7-item subscales (0-21 scale: 0-7 'normal', 8-10 'mild', 11-14 'moderate' and 15-21 'severe'). Higher scores indicated poorer emotional well being. ${ }^{10,11}$

The WPAI is a six-item measure used to quantify absenteeism, presenteeism and daily activity impairment customized to SCI-NeP. Scores were multiplied by 100 and expressed as impairment percentages. Higher values indicate worse outcomes. $^{12}$

\section{Costing algorithms}

Costing algorithms assigned 'per unit' costs (2012 US\$) to HRU, excluding subject-reported out-of-pocket expenses, which were used as reported and did not require monetization. Office visits, procedures and tests were based on the fiscal year (FY) 2012 Medicare Physician Fee Schedule. Hospital outpatient and emergency room visits were based on the FY 2012 Medicare Hospital Outpatient Prospective Payment System. Discharge diagnosis, procedures and length of stay mapped hospital admissions to appropriate Diagnosis Related Groups to calculate unit costs using the FY 2012 Medicare Hospital Inpatient Prospective Payment System. Redbook 2012 was used to identify unit costs of prescribed medications. Discounted average wholesale price plus a dispensing fee was used to cost prescription medications. ${ }^{13}$

Direct out-of-pocket costs to the subject for SCI-NeP, based on 4-week data, were multiplied by 13 to calculate annualized ( 52 week) per subject costs.

Indirect costs from work-related lost productivity were calculated using the 2011 average hourly wage values obtained through the Bureau of
Labor Statistics ${ }^{14}$ multiplied by the overall work impairment percentage from the WPAI. ${ }^{15}$ Indirect costs for lost productivity for subjects unemployed, retired early or with reduced work schedules due to SCI-NeP were calculated by multiplying average hourly wage by time since change in employment status due to SCI-NeP. Indirect costs for lost productivity for subjects disabled due to SCI-NeP were calculated using the average hourly wage plus the average monthly disability payment from the Social Security Administration. ${ }^{16}$

Annualized costs of SCI-NeP per subject were calculated as follows: direct medical cost to payers, direct costs to subjects and indirect costs.

\section{Statistical methods}

Summary statistics (means and s.d. for continuous variables and frequency distributions for categorical variables) were used to describe the sample. BPI average pain severity scores were used to classify pain severity (0-3 mild, 4-6 moderate and $7-10$ severe). ${ }^{17,18}$ To evaluate the association between pain severity categories and other outcomes, Kruskal-Wallis test was used for continuous variables, and $\chi^{2}$-test or Fisher's exact tests for categorical variables.

The association between pain severity and costs was examined using multiple (adjusted) linear regression. For the adjusted model, stepwise regression was used with a pool of covariates: age, sex, race, ethnicity, pain severity, employment status, walking ability, insurance coverage, prescription coverage, worker's compensation, time since diagnosis and comorbid conditions

Statistical significance was evaluated at the 0.05 level. All analyses were performed using PC-SAS version 9.1.3 (SAS Institute, Cary, NC, USA).

\section{RESULTS}

\section{Demographic and clinical characteristics}

Table 1 presents demographic and clinical characteristics of the sample overall and by pain severity. Of the 103 SCI-NeP subjects enrolled, mean (s.d.) age was 48.7 (14.6) years, and 72 (69.9\%) were male. Mean pain severity score was 5.3 (2.1) overall (mild: 2.3 (1.0), moderate: $5.2(0.7)$ and severe: $7.9(1.0)$ ), with $20.4 \%$ of subjects experiencing mild, $51.5 \%$ moderate and $26.2 \%$ severe pain. Mean time since SCI-NeP diagnosis was 7.6 (6.9) years. Mean time from appearance of SCI-NeP symptoms to time of diagnosis was 2.8 years. The course of pain was most frequently described (44.7\% of subjects) as 'persistent pain with pain attacks', and the majority of subjects reported having a tingling or prickling sensation in the area of their pain $(84.6 \%)$ and/or suffering from a burning sensation $(59.2 \%)$ (responses ranged from slight to very strong) (data not shown). Subjects had an average of 2.2 (1.5) comorbidities; the most common were sleep disturbance/insomnia (28.2\%), depressive symptoms $(25.2 \%)$ and anxiety (23.3\%) (Figure 1 ). Only half of SCI-NeP subjects $(51.5 \%)$ were able to walk on their own.

\section{Health-related quality-of-life}

The mean SF-12 Physical Component Summary (PCS) and Mental Component Summary (MCS) scores were 30.8 (7.5) and 45.8 (13.4), respectively (Figure 2a). The following SF-12 domain scores were significantly lower among subjects with greater pain severity: bodily pain, general health and vitality $(P<0.035)$ (data not shown). The mean EQ-5D health utility was $0.49(0.25)$ and decreased significantly as pain severity increased $(P=0.0004)$ (Figure $2 \mathrm{~b}$ ).

Mean BPI-SF pain interference index was 5.4 (2.3) and increased significantly as pain severity increased $(P<0.0001)$ (Figure $2 \mathrm{c}$ ). Mean MOS Sleep Problems Index score was 46.9 (21.8) and worsened significantly as pain severity increased $(P=0.0034)$ (Figure $2 \mathrm{~d}$ ). Across pain severity levels, over half $(59.2 \%$ overall) of the SCI-NeP subjects experienced some level of anxiety, with a mean HADS anxiety score of 8.4 (3.8) overall (data not shown). Among moderate and 
Table 1 Demographic and clinical characteristics, overall, by average pain severity

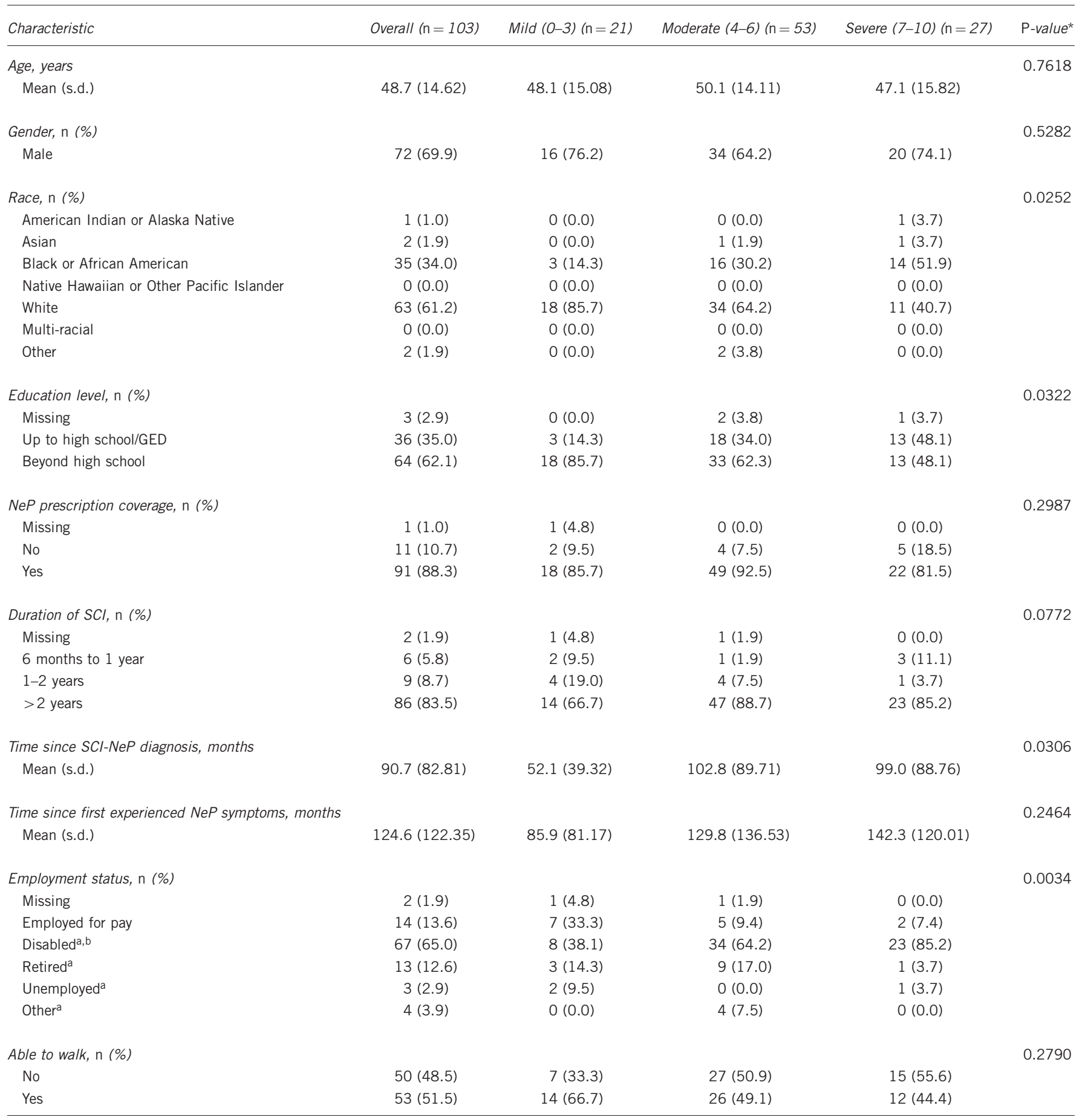

Abbreviations: GED, general educational diploma; NeP, neuropathic pain; SCI, spinal cord injury.

* P-values are from the Kruskal-Wallis test for continuous variables, and the Fisher's exact test for the categorical variables; mild vs moderate vs severe.

Note: Mild, moderate and severe classification was based on the BPI average pain severity score. Two subjects did not respond to all required items needed to calculate a BPI average pain severity score and thus were not included in any analysis by pain severity category.

As more than one response may be selected, the sum of percentages across response options may exceed 100

aAmong respondents not employed for pay.

boverall, 23 out of 67 reported being disabled due to their SCI-NeP ( 4 of 8 mild, 13 of 34 moderate and 6 of 23 severe).

severe pain groups, more than half $(51.0 \%$ and $51.8 \%$, respectively) experienced some level of depression; overall and in the mild pain group, a smaller proportion ( $45.7 \%$ and $28.5 \%$, respectively) experienced some level of depression. Mean HADS depression score was 7.2 (4.5) (data not shown).

\section{Health resource use}

Most (94.2\%) subjects were prescribed $\geqslant 1$ SCI-NeP medication, including $100 \%$ of subjects (27) with severe pain (data not shown). One-third $(32.1 \%)$ of subjects were prescribed $\geqslant 3$ medications for their SCI-NeP, with an average of 2.2 (1.6) prescriptions per subject 


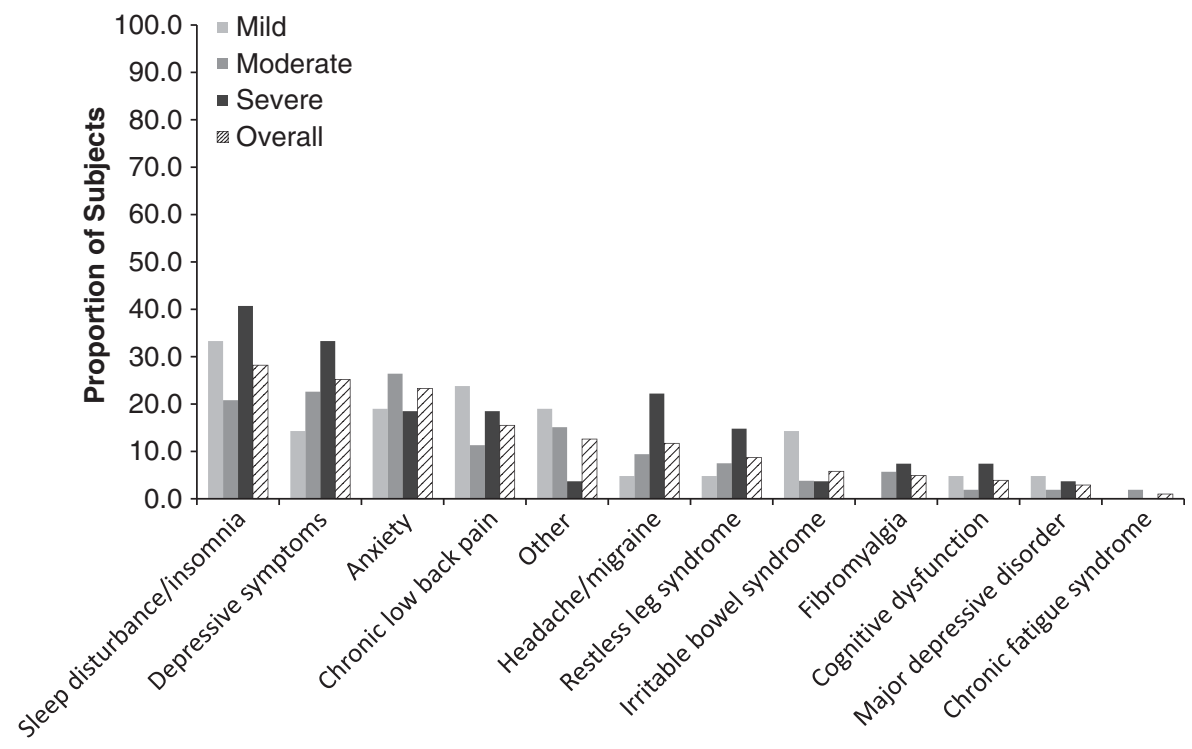

Figure $1 \mathrm{SCl}-\mathrm{NeP}$ subjects reported a variety of comorbid conditions.
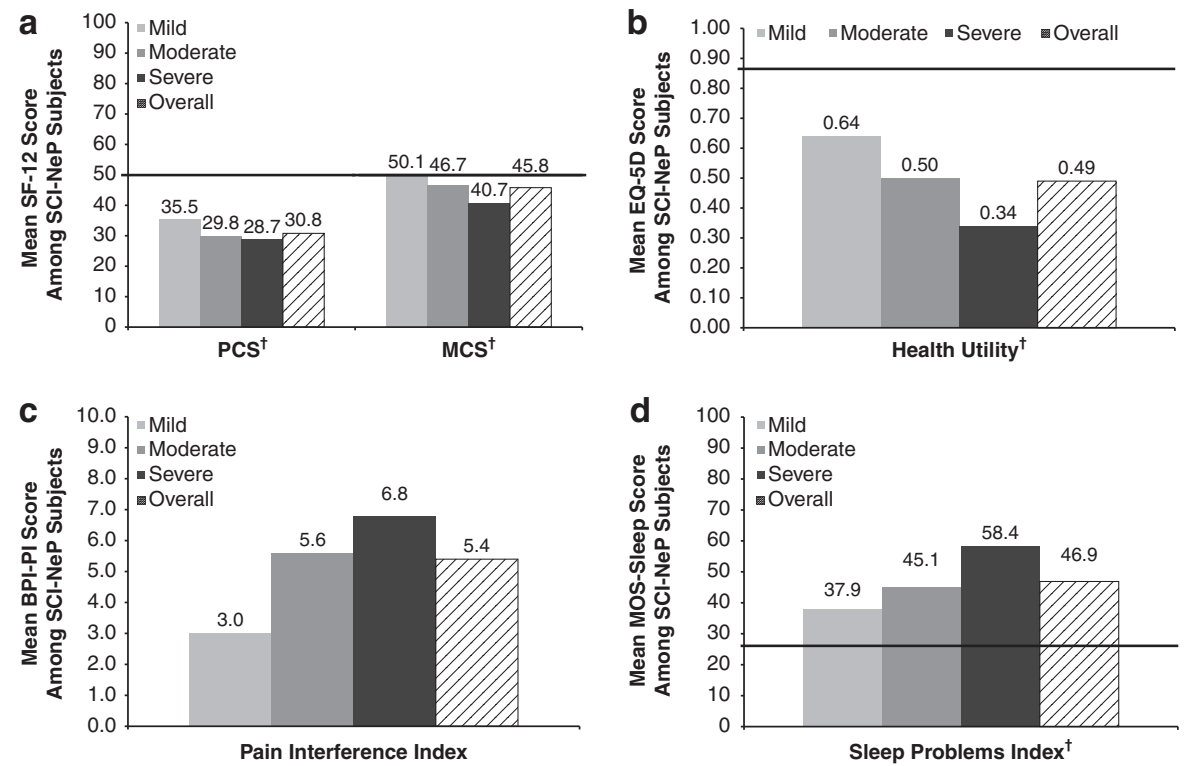

Figure 2 (a) SCl-NeP subjects with more severe pain had worse physical and mental health status (scores on the BPI pain severity were used to classify average pain severity. No significant differences were observed across pain severity levels). "Population norms (PCS $=49.7$ and $M C S=49.5)$ indicated by thick black horizontal lines. (b) General health status significantly worse among SCI-NeP subjects with more severe pain (scores on the BPI pain severity were used to classify average pain severity. Mean EQ-5D health-state utilities measured on -0.11 to 1.00 scale. A significant difference was observed across pain severity levels for health-state utilities $(P=0.0004)$. †Population norm $(0.87)$ indicated by thick black horizontal line). (c) SCl-NeP subjects with more severe pain had greater pain interference with function (scores on the BPI pain severity were used to classify average pain severity. BPI pain interference index scored on a $0-10$ scale. A significant difference was observed across pain severity levels for BPI pain interference index $(P<0.0001)$. Note: All domains (general activity, mood, walking ability, normal work, relations with other people, sleep and enjoyment of life) were significantly different by pain severity $(P<0.0033)$ ). (d) SCl-NeP subjects with more severe pain had significantly worse sleep outcomes (scores on the BPI pain severity were used to classify average pain severity. MOS Sleep Problems Index scored on a 0-100 scale. A significant difference was observed across pain severity levels for the index score $(P=0.0034){ }^{\dagger}$ Population norm (25.8) indicated by thick black horizontal line).

(Table 2). The most commonly prescribed medication classes were antiepileptics (64.1\%), strong short-acting opioids (34.0\%) and longacting opioids $(23.3 \%)$ (Figure 3 ). The most commonly prescribed antiepileptics were gabapentin $(75.8 \%)$ and pregabalin $(25.8 \%)$ (data not shown). The most commonly prescribed strong short-acting opioids were oxycodone/acetaminophen $(45.7 \%)$ and oxycodone hydrochloride immediate release $(40.0 \%)$ (data not shown). The most commonly prescribed long-acting opioids were morphine sulfate-extended release (54.2\%) and methadone (37.5\%) (data not shown).

Nearly half $(44.7 \%)$ of the subjects reported taking non-prescription medications for their SCI-NeP in the 4 weeks before enrollment, including ibuprofen (20.4\%) and acetaminophen (12.6\%) (data not shown). 
Table 2 Resource utilization for $\mathrm{SCl}-\mathrm{NeP}$, overall, by average pain severity

\begin{tabular}{|c|c|c|c|c|c|}
\hline Resource use & Overall $(\mathrm{n}=103)$ & Mild $(0-3)(n=21)$ & Moderate $(4-6)(n=53)$ & Severe $(7-10)(n=27)$ & P-value* \\
\hline \multicolumn{6}{|l|}{ Medication } \\
\hline Prescription medications prescribed to subjecta, mean (s.d.) & $2.2(1.55)$ & $1.7(1.27)$ & $2.3(1.59)$ & $2.4(1.67)$ & 0.2901 \\
\hline Non-prescription medications used ${ }^{\mathrm{b}}$, mean (s.d.) & $0.9(1.13)$ & $0.7(1.10)$ & $1.0(1.18)$ & $0.9(1.12)$ & 0.6151 \\
\hline \multicolumn{6}{|l|}{ Office visit ${ }^{\text {a }}$} \\
\hline Physician office visits for $\mathrm{SCl}-\mathrm{NeP}$, mean (s.d.) & $2.2(2.09)$ & $2.1(2.17)$ & $2.5(2.26)$ & $1.7(1.59)$ & 0.2873 \\
\hline Non-physician office visits for SCl-NeP, mean (s.d.) & $0.0(0.00)$ & $0.0(0.00)$ & $0.0(0.00)$ & $0.0(0.00)$ & 1.0000 \\
\hline \multicolumn{6}{|l|}{ Test and procedure ${ }^{\mathrm{a}}$} \\
\hline Outpatient tests or procedures, mean (s.d.) & $0.4(1.14)$ & $0.9(1.51)$ & $0.3(1.18)$ & $0.2(0.48)$ & 0.0181 \\
\hline ER visits for SCl-NePa, mean (s.d.) & $0.0(0.14)$ & $0.0(0.00)$ & $0.0(0.14)$ & $0.0(0.19)$ & 0.6598 \\
\hline Hospital outpatient visits for SCl-NePa, mean (s.d.) & $0.0(0.10)$ & $0.0(0.00)$ & $0.0(0.00)$ & $0.0(0.19)$ & 0.2540 \\
\hline Hospitalizations for $\mathrm{SCl}-\mathrm{NePa}^{\mathrm{a}}$ mean (s.d.) & $0.0(0.10)$ & $0.0(0.00)$ & $0.0(0.00)$ & $0.0(0.19)$ & 0.2540 \\
\hline
\end{tabular}

Abbreviations: ER, emergency room; SCI-NeP, spinal cord injury-related neuropathic pain.

* $P$-values are from the Kruskal-Wallis test for continuous variables; mild vs moderate vs severe.

Note: Mild, moderate and severe classification was based on the BPI average pain severity score. Two subjects did not respond to all required items needed to calculate a BPI average pain severity score and thus were not included in any analysis by pain severity category.

a Over the past 6 months.

bover the past 4 weeks.

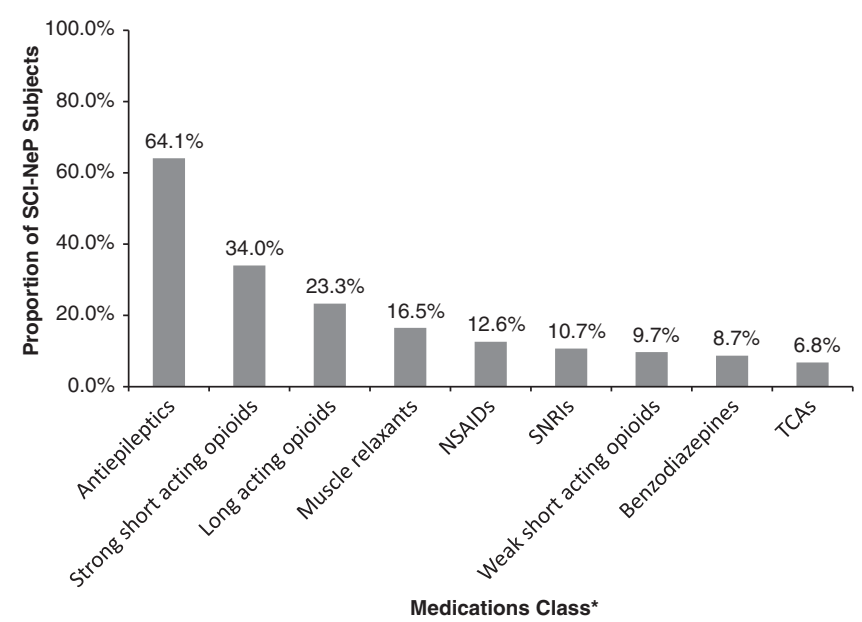

Figure $3 \mathrm{SCl}-\mathrm{NeP}$ prescription medication use. *Figure includes all reported classes with at least $2 \%$ of subjects prescribed one or more medications in the class in the past 6 months, excluding miscellaneous agents.

Mean number of SCI-NeP-related physician office visits per subject in the prior 6 months was 2.2 (2.1) (Table 2). Mean number of officebased tests and procedures performed in the 6 months prior was 0.4 (1.1), with significantly fewer tests and procedures performed on subjects with more severe pain $(P=0.0181)$. There were two SCI-NeP-related emergency room visits, one SCI-NeP-related hospital outpatient visit and one SCI-NeP-related hospitalization.

\section{Employment and lost productivity}

Few $(13.6 \%)$ subjects were employed for pay, and the proportion significantly declined as pain severity increased $(P=0.0034)$. Onequarter of $(22.3 \%)$ subjects were disabled due to their SCI-NeP, and most $(82.6 \%)$ had moderate or severe pain (Table 1). Among subjects employed for pay, mean overall work impairment was 38.0\% (28.5\%). Across all subjects, mean overall activity impairment was $54.1 \%$ $(26.3 \%)$, which increased/worsened with increased pain severity $(P<0.0001)$ (Table 3).
Costs

Unadjusted mean (95\% confidence interval) annualized direct cost per subject was $\$ 8636$ (\$6176, \$11096) and increased as pain severity increased (mild: \$5777, moderate: $\$ 8576$ and severe: \$11492; $P=0.2996)$. The largest proportion was for prescription drugs (50.3\%) followed by out-of-pocket medical costs to subjects $(18.1 \%)$ and office-based tests and procedures (12.7\%). Unadjusted mean annualized indirect cost per subject was $\$ 17634$ (\$12 845, \$22 422) overall and was highest among those with moderate and severe pain (mild: \$16 943, moderate: $\$ 19888$ and severe: $\$ 15052 ; P=0.6957$ ).

Results from the regression analysis showed statistically significant differences in direct and indirect costs per subject across pain severity levels $(P<0.0001)$ (Figure 4$)$. Total mean annualized adjusted direct costs per subject were $\$ 7940$ for mild, $\$ 7630$ for moderate and $\$ 11666$ for severe. Total mean annualized adjusted indirect costs per subject were \$19492 for mild, \$17559 for moderate and \$17 642 for severe. Total mean (95\% confidence interval) annualized adjusted direct and indirect costs per subject overall remained similar to the unadjusted results: $\$ 8636$ (\$6604, \$10668) and \$17634 (\$13527, $\$ 21741$ ), respectively.

\section{DISCUSSION}

This is the first study to comprehensively evaluate the burden of SCI-NeP among US adults. Subjects were required to have a diagnosis of SCI-NeP for at least 6 months to capture HRU and were actively seeking care. The study results may be used to inform physician and patient dialog regarding appropriate treatment of pain and other important outcomes among this population (for example, sleep, anxiety and depression).

An association between pain severity and subject-reported health status was evident. Health status decreased significantly as pain severity increased. Although subjects were actively managed, SCI$\mathrm{NeP}$ subjects experienced substantially lower health status than the general US population, as reflected by scores on the PCS and MCS (Figure 2a) and utilities (Figure 2b) compared with the US normative values of 49.5, 49.7 and 0.87 , respectively. ${ }^{19,20}$ Those with severe pain reported the worst health status, which was particularly evident when comparing utilities to normative values ( 0.34 vs 0.87$)$. A high 
Table 3 Work productivity and activity impairment: SCI-NeP (WPAI:SCI-NeP), overall, by average pain severity

\begin{tabular}{|c|c|c|c|c|c|}
\hline Productivity & Overall $(n=103)$ & Mild $(0-3)(n=21)$ & Moderate $(4-6)(n=53)$ & Severe $(7-10)(n=27)$ & P-value* \\
\hline Overall work impairment ${ }^{a, b}$ & & & & & 0.3476 \\
\hline Mean (s.d.) & $38.0(28.45)$ & $25.8(20.79)$ & $50.7(34.59)$ & $60.0(\mathrm{~N} / \mathrm{A})$ & \\
\hline Median & 40.0 & 10.0 & 66.7 & 60.0 & \\
\hline Range & $0-80$ & $10-60$ & $0-80$ & $60-60$ & \\
\hline$n$ & 100 & 21 & 52 & 25 & \\
\hline Mean (s.d.) & $54.1(26.33)$ & $30.5(24.18)$ & $54.8(22.27)$ & $73.6(19.55)$ & \\
\hline Median & 60.0 & 30.0 & 60.0 & 70.0 & \\
\hline Range & $0-100$ & $0-100$ & $0-100$ & $20-100$ & \\
\hline
\end{tabular}

Abbreviations: N/A, not applicable; NeP, neuropathic pain; SCl, spinal cord injury.

${ }^{*} P$-values are from the Kruskal-Wallis test for continuous variables, and the Fisher's exact test for categorical variables; mild vs moderate vs severe.

Note: Mild, moderate and severe classification was based on the BPI average pain severity score. Two subjects did not respond to all required items needed to calculate a BPI average pain severity score and thus were not included in any analysis by pain severity category.

Note: The overall work impairment score is based on subjects who provided a value for presenteeism and/or absenteeism unless all values were ' 0 '.

a Higher values indicate greater impairment.

${ }^{\mathrm{b}}$ Among those employed for pay.

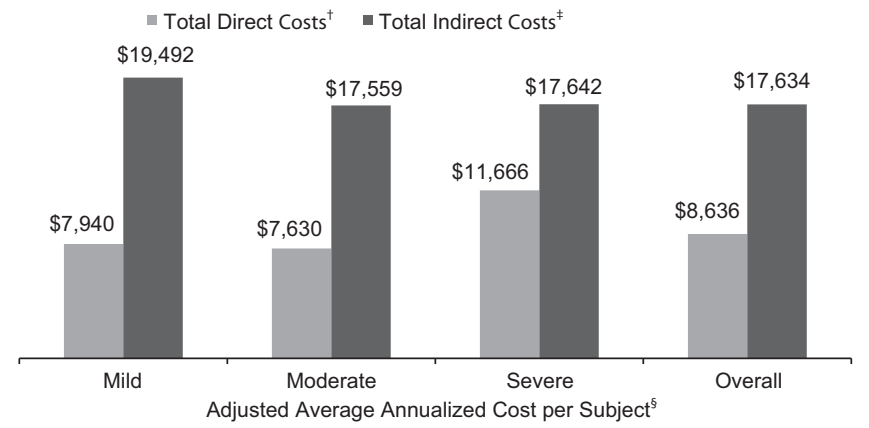

Figure 4 Adjusted average annualized cost per SCI-NeP subject, overall and by average pain severity (scores on the BPI pain severity were used to classify average pain severity as follows: $0-3$ represents mild pain, 4-6 represents moderate pain and $7-10$ represents severe pain. ${ }^{\dagger}$ Average annualized total direct cost per subject was significantly different by pain severity $(P<0.0001)$. Direct costs include physician visits, other healthcare provider visits, prescription medications, transcutaneous electrical nerve stimulation device, outpatient tests/procedures, emergency room visits, hospital outpatient visits, hospitalizations, direct medical costs to subjects and direct non-medical (child care, help with house and/or yard work and help with activities of daily living) due to SCl-NeP. ${ }^{\ddagger}$ Average annualized total indirect cost per subject was significantly different by pain severity $(P<0.0001)$. Total indirect costs include overall work impairment, activity impairment, disability, unemployment, early retirement and reduced work schedule due to SCI-NeP. \$Adjusted least squares mean estimates from multiple linear regression adjusted for confounding demographic and clinical variables. Specifically, covariates for direct costs: sex, pain severity (mild/moderate/severe only), time since diagnosis and comorbidities (headache/migraine and fibromyalgia); for and indirect costs: age, sex, pain severity (mild/moderate/severe only), and comorbidities (restless leg syndrome, cognitive dysfunction, chronic fatigue syndrome, anxiety and other).

proportion of subjects were prescribed opioids and NSAIDS; their position as 'first-line' pharmacological agents for $\mathrm{NeP}$ is controversial. These results point to the potential humanistic benefits of more effective management of SCI-NeP.

Sleep outcomes were worse among subjects with greater pain severity. In comparison with the US normative data on the MOS Sleep Problems Index, ${ }^{9}$ SCI-NeP patients had substantially higher scores, indicating worse sleep outcomes (Figure 2d). Consistent with previous research of post-SCI pain, ${ }^{3-5}$ these findings provide additional evidence that effective management of SCI-NeP subjects should address sleep outcomes.

High HRU among SCI-NeP subjects resulted in substantial total direct medical costs per subject, including high out-of-pocket costs to subjects. Indirect costs due to SCI-NeP accounted for the majority of total costs, and were driven by changes in employment status, as few subjects were employed for pay and almost one-quarter of subjects were disabled due to SCI-NeP. SCI-NeP subjects experience not only high humanistic burden but also high economic burden, and the economic burden affects payers and employers as well.

\section{Limitations}

There are some limitations inherent with this study. The study enrolled subjects actively seeking medical care for their SCI-NeP. Further, this study enrolled subjects diagnosed with SCI-NeP at least 6 months ago who had been managed at the physician's practice for the same time period. As such, these findings may not be generalizable to others with SCI-NeP who are not seeking treatment or do not regularly visit their physician.

This study required a retrospective review of medical records, which likely led to under-reporting of HRU. The subject's medical record may not include all visits to other physicians, health-care providers or facilities, including SCI-NeP-related tests and procedures, and medications prescribed outside of the study site. One interesting aspect of this study design was its focus on collecting HRU specifically attributable to SCI-NeP, compared with study designs, such as claim analysis, which assess all-cause HRU among SCI-NeP subjects, and, thus, may capture HRU attributable to the underlying SCI.

Costs were assigned to HRU using standard algorithms, which may have over- or underestimated costs. Finally, lost productivity and outof-pocket costs were based on subject recall, and may have resulted in over- or underestimation.

\section{CONCLUSION}

The majority of subjects with SCI-NeP in this study reported moderate and severe levels of pain, on average, and sub-optimal 
levels of overall health, functioning and well being, despite receiving active management. Outcomes worsened among subjects with higher pain severity. Further, the economic burden of SCI-NeP, particularly indirect costs, was substantial. The impact on quality-of-life and costs attributable to SCI-NeP highlight the unmet need and the potential benefit of more effective management of SCI-NeP to patients, payers and society.

\section{DATA ARCHIVING}

There were no data to deposit.

\section{CONFLICT OF INTEREST}

Alesia Sadosky and Bruce Parsons are paid employees of Pfizer, Inc. Rebecca Baik, Felicia Bergstrom, Rachael Mann and Caroline Schaefer are employees of Covance Market Access Services Inc, who were paid consultants to Pfizer, Inc in the development and execution of this study and manuscript. Drs Alan Anschel and Srivinas Nalamachu were paid investigators for the study, but were not financially compensated for their publication-related activities. Drs Brett Stacey, Edward Nieshoff and Michael Tuchman were not financially compensated for their collaborative efforts on this project, including publication-related activities.

\section{ACKNOWLEDGEMENTS}

We thank Gergana Zlateva, PhD, and Shoshana Daniel, PhD, for their contributions to the study design and analysis.

1 Siddall PJMJ, Rutkowski SB, Cousins MJ. A longitudinal study of the prevalence and characteristics of pain in the first 5 years following spinal cord injury. Pain 2003; 103 : 249-257.

2 Baastrup C, Finnerup NB. Pharmacological management of neuropathic pain following spinal cord injury. CNS Drugs 2008; 22: 455-475.

3 Raichle KAOT, Jensen MP, Cardenas D. The reliability and validity of pain interference measures in persons with spinal cord injury. J Pain 2006; 7: 179-186.
4 Turner JCD, Warms C, McClellan C. Chronic pain associated with spinal cord injured: a community survey. Arch Phys Med Rehabil 2001; 82: 501-508.

5 Widerstrom-Noga EGF-CE, Yezierski R. Chronic pain after spinal injury: interference with sleep and daily activities. Arch Phys Med Rehabil 2001; 82: 1571-1577.

6 Cleeland CS, Ryan KM. Pain assessment: global use of the brief pain inventory. Ann Acad Med Singapore 1994; 23: 129-138.

7 Ware J Jr., Kosinski M, Keller SDA. 12-Item Short-Form Health Survey: construction of scales and preliminary tests of reliability and validity. Med Care 1996; 34: 220-233.

8 Rabin R, de Charro F. EQ-5D: a measure of health status from the EuroQol Group. Ann Med 2001; 33: 337-343.

9 Hays RD, Martin SA, Sesti AM, Spritzer KL. Psychometric properties of the Medical Outcomes Study Sleep measure. Sleep Med 2005; 6: 41-44.

10 Snaith RP, Zigmond AS. The hospital anxiety and depression scale. Br Med J (Clin Res Ed) $1986 ; 292: 344$.

11 Zigmond AS, Snaith RP. The hospital anxiety and depression scale. Acta Psychiatr Scand 1983; 67: 361-370.

12 Reilly MC, Zbrozek AS, Dukes EM. The validity and reproducibility of a work productivity and activity impairment instrument. Pharmacoeconomics 1993; 4: 353-365.

13 Reimbursement PaDM. Available from http://www.benefitdesignreport.com/PharmacyReimbursement/tabid/66/Default.aspx

14 Values BoLSAHW. Available from http://www.bls.gov/oes/current/oes_nat.htm\#00 0000

15 Lofland JH, Pizzi L, Frick KDA. Review of health-related workplace productivity loss instruments. Pharmacoeconomics 2004; 22: 165-184.

16 Disability SSAAM. Available from http://www.ssa.gov/policy/docs/quickfacts/stat_ snapshot/

17 Zelman DC, Dukes E, Brandenburg N, Bostrom A, Gore M. Classification of mild, moderate and severe pain due to diabetic peripheral neuropathy based on levels of functional disability. J Pain 2004; 5 (Suppl. 1), 114.

18 Zelman DC, Dukes E, Brandenburg N, Bostrom A, Gore M. Identification of cut-points for mild, moderate and severe pain due to diabetic peripheral neuropathy. Pain 2005; 115: 29-36.

19 Ware JEKM, Turner-Bowker DM, Sundaram M, Gandek B, Maruish ME. User's manual for the SF-12v2 Health Survey Second Edition. QualityMetric, Incorporated 2009.

20 Luo NJJ, Shaw JW, Feeny D, Coons SJ. Self-reported health status of the general adult US population as assessed by the EQ-5D and Health Utilities Index. Medical Care 2005; 43: 1078-1086.

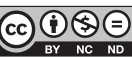

This work is licensed under a Creative Commons Attribution-NonCommercial-NoDerivs 3.0 Unported License. To view a copy of this license, visit http://creativecommons. org/licenses/by-nc-nd/3.0/ 